\title{
Der ökologische Indikatorwert mitteleuropäischer Pisidium-Arten (Mollusca, Eulamellibranchiata)
}

\author{
Von Claus Meier-Brook, Tübingen
}

Mit 2 Abbildungen

\begin{abstract}
$\mathrm{Z}$ u s a m m e n f a s s u n g. Eine Kurzübersicht über bisher bekannte ökologische Valenzen und Ansprüche der rezenten Muschelarten der Gattung Pisidium C. Pfeiffer in Mitteleuropa stellt Arten mit geringem Indikatorwert (z. B. P. subtruncatum, milium) solchen mit hohem Indikatorwert (z. B. P. supinum, P. lilljeborgii) gegenüber. Die Notwendigkeit, über bloße Präsenz hinaus die Dominanzverhältnisse zu berücksichtigen, wird betont.

$\mathrm{Su} \mathrm{m} \mathrm{m}$ a ry. The presently known ecological valences and requirements of recent species of pill-clams (Pisidium C. Pfeiffer) in Central Europe are briefly reviewed. Species of low indicator value (e. g. $P$. subtruncatum, milium) are contrasted with those having a high indicator value (e.g. $P$. supinum, lilljeborgii). The necessity of taking into account the abundance of species, rather than merely their presence, is emphasized.
\end{abstract}

\section{Einleitung}

Die Auswertung von Süßwassermolluskenfunden bei quartär-geologischen und vorgeschichtlichen Untersuchungen hat sich in vielen Fällen mehr oder weniger auf die Gastropoden beschränkt. Stenöke, also aussagekräftige, Arten sind unter ihnen jedoch spärlich, so daß die Kleinmuscheln der Familie Sphaeriidae immer mehr zur Lösung paläoökologischer Fragen mit herangezogen werden. Ein überzeugendes Beispiel stellt die Bearbeitung der Pisidien des ehemaligen Ascherslebener Sees dar (KUIPER, 1968). Die vagen Vorstellungen über den Aussagewert der einzelnen Arten, wie sie noch vor einigen Jahren bestanden, sind inzwischen einem präziseren Bild gewichen, wenn auch eine kausale Analyse der ökologischen Valenzen und Ansprüche noch lange auf sich warten lassen wird. Die Erfahrungen aus diskriptiv-ökologischen Untersuchungen an Hunderten Gewässern in den letzten Jahren lassen immerhin über Valenzen und Präferenzen deutliche Aussagen zu, die im folgenden niedergelegt werden. Die Darlegungen beschränken sich auf die rezent in Mitteleuropa lebenden Arten und ihre Ókologie unter gemäßigtem Klima. Über Pisidium astardoides SANDberger sowie P. stewarti Preston (meist unter dem Namen P. vincentianum WOODWARD) ist in der Literatur unverhältnismäßig viel berichtet worden. Ihre Bedeutung ist daher am bekanntesten. Eine Bestimmungstabelle in deutscher Sprache bringt ZeIssler (1971), jedoch sollte ohne langjährige Erfahrungen die Bestimmung unbedingt Spezialisten überlassen werden. Wegen der erheblichen Bestimmungsschwierigkeiten liegt jedoch manches wertvolle Material brach, das nach Identifikation durch Spezialisten wichtige Aufschlüsse geben könnte.

Es ist nicht Aufgabe dieses Artikels, die umfangreiche Literatur der letzten Jahre kritisch zusammenzufassen oder sie durch eine ausführliche Analyse eigener Untersuchungen zu ergänzen. Hier soll lediglich eine kurzgefaßte Übersicht gegeben werden, die dem Paläontologen einen schnellen Überblick über den ökologischen Indikatorwert seiner Pisidienausbeute ermöglichen möchte. Ausnahmen von den groben Eingruppierungen sind selbstverständlich möglich, vor allem unter stark abweichenden Klimabedingungen, und es muß 
betont werden, daß nie allein Präsenz oder Absenz, sondern immer auch Dominanzverhältnisse die Auswertung mitbestimmen sollten. Auf eine Benennung von Untergattungen wird angesichts der noch immer unklaren Abgrenzungen verzichtet.

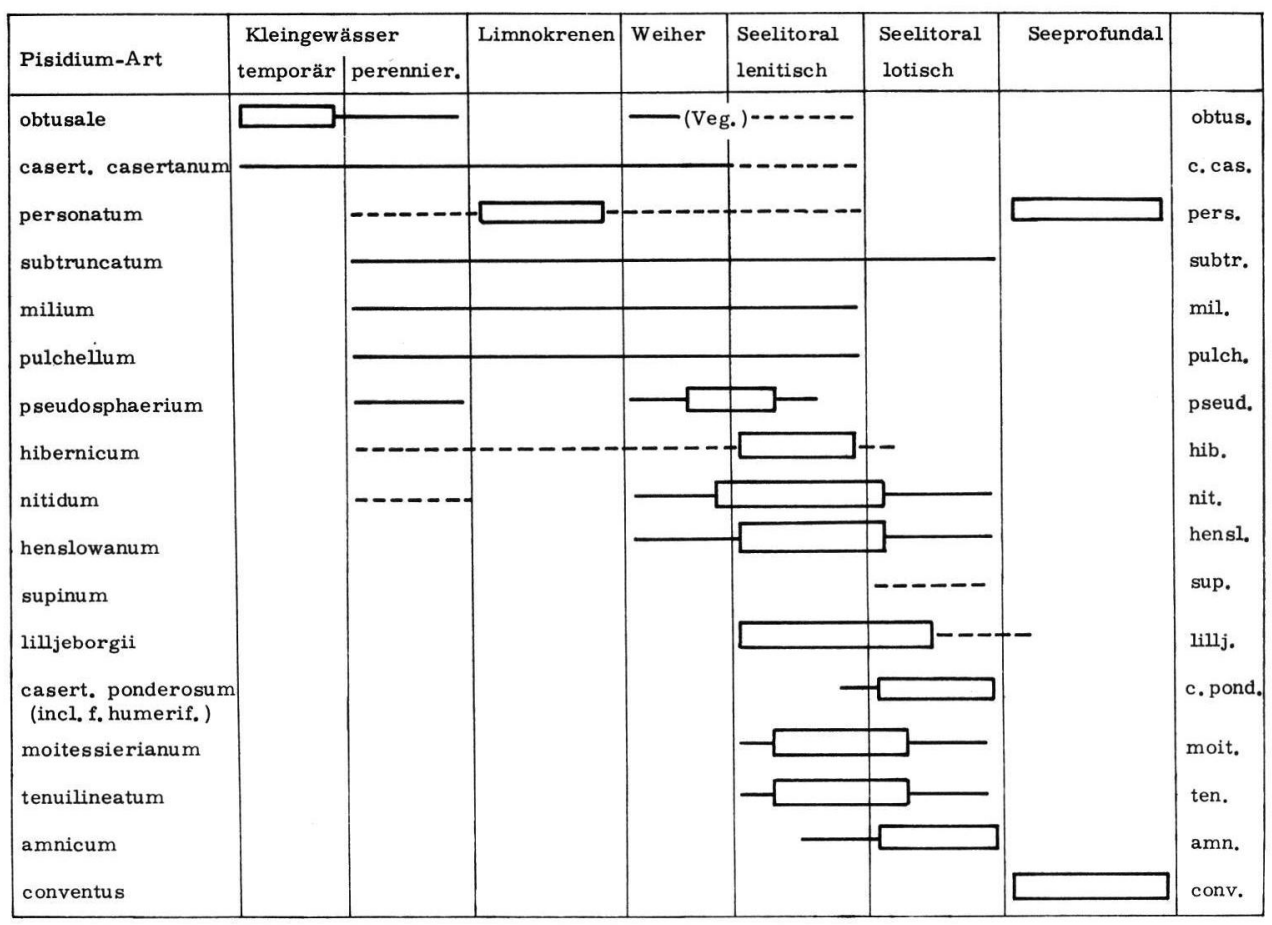

Abb. 1. Ókologische Verbreitung der Pisidien in s t e h e n d e n Gewässern. Gewässer nach Größe und Wasserbewegung geordnet. Angaben nur für gemäßigtes Klima.

$$
\text { Zeichenerklärung: } \begin{aligned}
& \square \text { deutliche Präferenz, hier im allg. dominant } \\
& \text { regelmäßig vorkommend }
\end{aligned}
$$

\section{Ökologische Charakterisierung der Arten}

Eine Übersicht des Vorkommens und der bevorzugten Biotope geben die Abbildungen 1 und 2, in denen die Gewässer nach Größe und Wasserbewegung angeordnet sind. Weitere Bemerkungen zu den Arten folgen in der in den Abbildungen gewählten Reihenfolge.

\section{Definitionen:}

E u ryther $\mathrm{m}$ : starken Temperaturschwankungen unterworfen bzw. diese tolerierend.

$\mathrm{K}$ a l k bedürftig: Nur bei Kalkgehalten über $5-7 \mathrm{mg} / 1$ (entsprechend $0,7-1^{\circ} \mathrm{dH}$ ), in diesem Bereich aber ohne weitere Abhängigkeiten. Nur kalkärmste Gewässer meidend.

$\mathrm{Ka}$ l k indifferent: Auch unterhalb $5-7 \mathrm{mg} / \mathrm{l}$ vorkommend.

( $\mathrm{K}$ a $1 \mathrm{~km}$ e ide $\mathrm{nde}$ Arten gibt es unter Pisidien im Gebiet nicht.)

L e n it is ch: mit ruhigem Wasser; besonders bezogen auf Stillwasserzonen in bewegten Gewässern. 


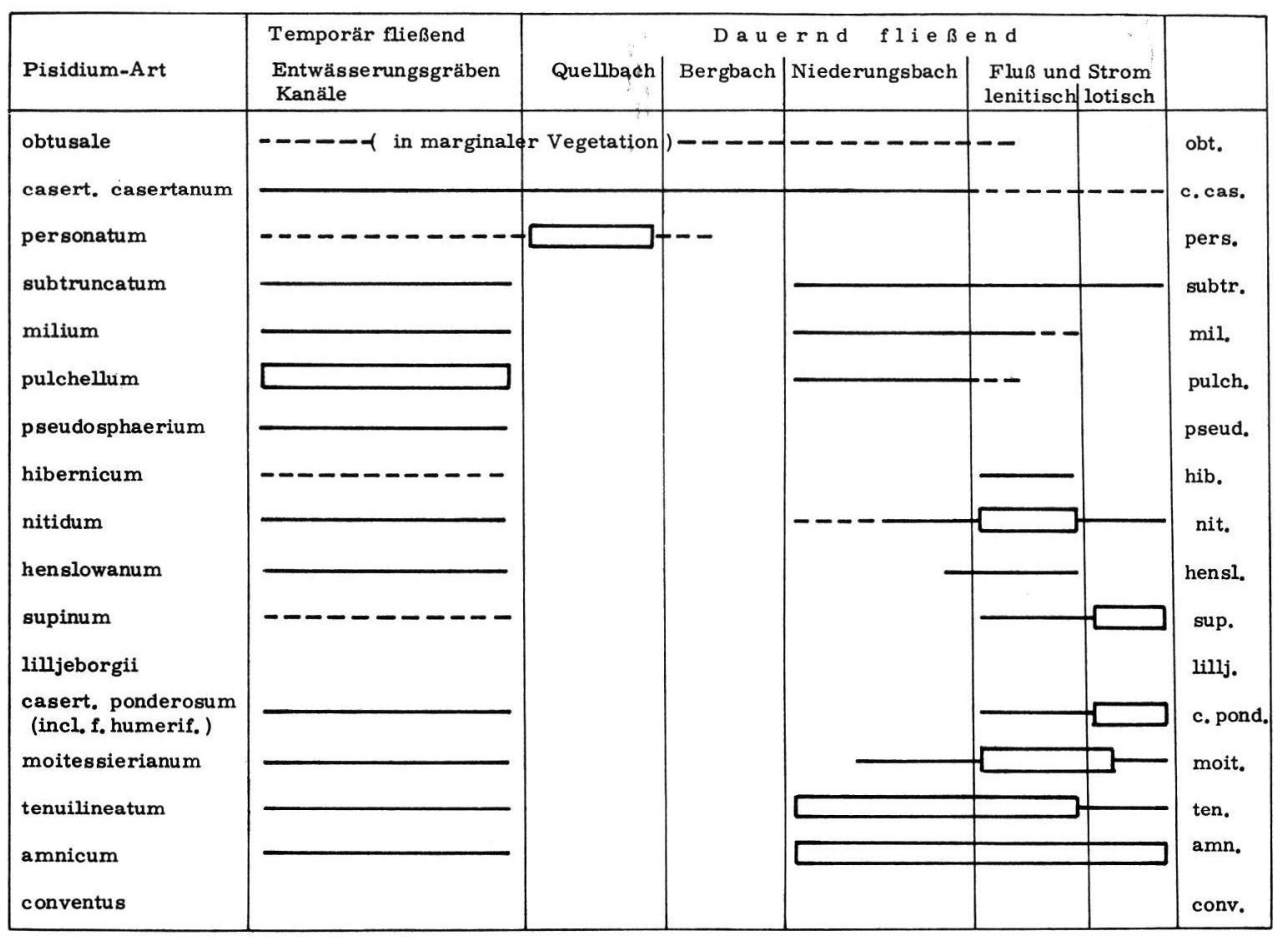

Abb. 2. Okologische Verbreitung der Pisidien in $\mathrm{fli}$ i $\mathrm{B}$ e $\mathrm{nden}$ Gewässern. Übrige Angaben wie bei Abb. 1.

L o t is ch : mit bewegtem Wasser (z. B. Brandungsufer, Stromschnellen).

Lim nokrene: Grundwasseraustritt (Quelle) am Boden eines im übrigen stehenden Gewässers.

$\mathrm{S}$ t e n o the r m : geringen Temperaturschwankungen unterworfen bzw. nur solche tolerierend.

\section{Pisidium obtusale (LAmarcK)}

Eine Moor- und Sumpfart, die humusreiche Gewässer bevorzugt (Dy). Sie ist das einzige Pisidium im Gebiet, das auf höhere Vegetation angewiesen erscheint, vor allem auf Hartflora (Cyperaceae, Juncaceae u. a.). Es lebt in zeitweise austrocknenden Tümpeln oft massenhaft und wird dort lediglich von $P$. casertanum begleitet. Kalkindifferent.

\section{P. casertanum casertanum (PoLI)}

In der Nominatrasse in einer Unzahl von Formen in einem weiten ökologischen Bereich vorkommend, ist es dennoch charakteristisch vor allem für Kleingewässer. Im Seelitoral und in Flüssen tritt es höchstens vereinzelt auf und wird in deren bewegten Zonen von P. casertanum ponderosum ersetzt. Es kann unter extremen Bedingungen existieren, was Nahrungsarmut, Kalkarmut, Austrocknungsmöglichkeiten und Temperaturschwankungen betrifft. Kommt mit einem Minimum an Schlamm aus. Kalkindifferent.

\section{$P$. personatum Malm}

Bevorzugt eindeutig gleichmäßig kühle Biotope, und zwar subterrane Gewässer, Quellen und Quellbäche, Limnokrenen sowie das Profundal tiefer Seen, solange das Wasser 
genügend Kalk und Sauerstoff enthält. In eurythermen Gewässern möglicherweise auf die Nähe litoraler Grundwasseraustritte beschränkt. Kalkbedürftig.

\section{P. subtruncatum MALM}

Das am meisten euryöke der mitteleuropäischen Pisidien. Soweit ein gewisser Schlammanfall gewährleistet ist, kommt es in allen Gewässertypen regelmäßig vor. Kalkindifferent.

\section{P. milium HeLD}

Die Art ist in allen schlammreichen Biotopen verbreitet, jedoch fast nirgends in hoher Abundanz. Kalkindifferent.

\section{P. pulchellum JENYNS}

Ein ausgesprochener Sumpfbewohner mit Präferenz für dunklen Schlamm; meidet moorige Gewässer. Kalkbedürftig.

\section{P. pseudosphaerium SCHLESCH}

Ein Flachmoorbewohner, charakteristisch für Verlandungszonen größerer Gewässer. Oft im Schwingrasen, nicht in Hochmooren. Kalkbedürftig.

\section{P. hibernicum WESTERLUND}

Im schlammreichen Sediment von Seen und Flüssen, aber auch in kleinen Moorgewässern, oft im Schwingrasen. Regelmäßig in Seen der Vereisungsgebiete. Kalkindifferent.

\section{$P$. nitidum JENYNS}

In allen Gewässern außer Quellen, Bächen und Tümpeln. Deutliche Präferenz aber für Großgewässer wie Seen und Tieflandflüsse, wo die Art in Mitteleuropa meist nicht nur die größte Abundanz aller Pisidium-Arten aufweist, sondern oft auch mehr als die Hälfte aller Kleinmuschelindividuen stellt. In schlammreichem Boden im allgemeinen zartschalig, in sandigem Boden lotischer Bezirke dickschalig und oft stark gerippt (f. crassa STELFox und f. arenicola StELFOX). Kalkindifferent.

\section{P. henslowanum (SHEPPARD)}

In allen Großgewässern mit einem gewissen Mindestkalkgehalt, auch mit (nicht zu heftiger) Wasserbewegung. Nicht in Bächen und Tümpeln. Kalkbedürftig.

\section{P. supinum A. ScHMIDT}

Die am strengsten auf stark bewegtes Wasser beschränkte Art. Fast nur in großen Flüssen mit stärkerer Strömung, bisweilen in stark lotischen Seezonen. Nie außerhalb dieser beiden Biotope. Kalkbedürftig.

\section{P. lilljeborgii CLESSIN}

Die in Mitteleuropa neben $P$. conventus am stärksten stenöke Art. Sie kommt ausschließlich im Litoral und Sublitoral von Seen vor. Kalkindifferent.

\section{P. casertanum ponderosum STELF. (incl. f. humeriforme STELF.)}

Diese vom Verfasser als ökologische Rasse von P. casertanum (PoLI) aufgefaßte Form ist ähnlich wie $P$. supinum auf stark bewegtes Wasser beschränkt, aber gleichermaßen in Flüssen wie Seen. Kalkbedürftig. 


\section{P. moitessierianum PALADILHE}

Lebt in Flüssen und Seen und scheint dort als ausgesprochener Thanatozönosen-(„Schalenzonen“-)Bewohner aufzutreten. Zumindest war in 4 untersuchten Seen und einem Fluß, in denen diese Art regelmäßig auftritt, unter Tausenden leerer Muschelschalen verschiedener Arten Tiere von P. moit. neben vereinzelten von P. nitidum die einzigen lebenden Pisidien. Die Art tritt hier oft mit beträchtlicher Abundanz auf. Kalkbedürftig.

\section{P. tenuilineatum STELFOX}

Im gleichmäßig strömenden Wasser von Tieflandbächen, Flüssen sowie im Litoral kalkreicher Seen. Bei der Seltenheit der Art kann die Frage, ob ihre Lebensweise ähnlich der von P. moitessierianum ist, noch nicht beantwortet werden. Kalkbedürftig.

\section{P. amnicum (O. F. MÜLleR)}

In bewegten Zonen von Flüssen und Seen, oft auf Sandboden mit geringsten Schlammbeimengungen. Kalkbedürftig.

\section{P. conventus Clessin}

Auf das Profundal tiefer Seen beschränkt. Kalkindifferent. Da diese Art in kalkärmstem, also mangelhaft gepuffertem Wasser lebt, werden die zarten Schalen nach dem Absterben schnell aufgelöst. $P$. conventus ist daher praktisch nie fossil erhalten. Kalkindifferent.

\section{Schlußfolgerungen auf den Indikatorwert}

In hoher Abundanz vorkommende Vertreter der Gattung lassen Schlüsse auf den Lebensraum entsprechend der Weite ihrer ökologischen Valenz nur sehr beschränkt zu im Falle folgender euryöker Arten: P. subtruncatum, milium, casertanum casertanum. Für Kleingewässer moorigen (dystrophen) Charakters spricht $P$. obtusale. Entweder auf quellnahe Biotope oder das Profundal kalkreicher Seen läßt $P$. personatum schließen.

Beim dominanten Vorkommen von $P$. nitidum ist an Gewässer einer Mindestgröße von Weiher (rezent auch Teich) oder Tieflandfluß zu denken, ebenso bei $P$. henslowanum. Die Anwesenheit von P. lilljeborgii ist eindeutiger Anzeiger für einen Seebiotop, es sei denn, es hätten zur Zeit der Ablagerung arktische oder subarktische Verhältnisse geherrscht.

Stark bewegte Zonen größerer Gewässer haben wir vor uns, wenn P. amnicum und $P$. casertanum ponderosum in ausreichender Häufigkeit angetroffen werden. Kommt $P$. supinum hinzu, spricht dies mit großer Wahrscheinlichkeit für einen Fluß. Stillere Zonen bewegter Gewässer zeigt $P$. moitessierianum an, möglicherweise auch $P$. tenuilineatum.

Funde von $P$. pseudosphaerium deuten auf einen Verlandungsprozeß (Flachmoorbildung) eines Sees oder Flußaltarms hin.

Die ökologischen Ansprüche einiger Arten, wie P. hibernicum und P. pulchellum, erscheinen bisher so heterogen und damit schwer faßbar, daß ihr Aussagewert noch gering anzusetzen ist. Im allgemeinen wird P. hibernicum in größerer Abundanz für einen See sprechen; die rezenten Funde in Flüssen und Kleingewässern sind verhältnismäßig selten.

Über häufige Vergesellschaftungen liegen mehrere Publikationen aus den letzten Jahren vor, deren Studium für weitergehende Beschäftigung empfohlen wird. Einige sind im Literaturverzeichnis genannt. Die umfangreiche Literatur mit Einzelangaben zur ökologischen Valenz und Präferenz kann hier nicht aufgeführt werden. Es sei lediglich auf das Literaturverzeichnis bei ỚKLAND (1971) hingewiesen. 
Für freundliche Durchsicht des Manuskripts und Anregungen danke ich den Herren J. G. J. Kuiper, Paris, Dr. K. Münzing, Freiburg, und Frau Dr. H. Zeissler, Leipzig.

\section{Literatur}

Bishop, M. J. \& Hewitt, S. J. (im Druck): Assemblages of Pisidium species (Bivalvia, Sphaeriidae) from localities in eastern England. Freshwater Biology, Oxford [u. a.].

Combes, C., Kuiper, J. G. J. \& StÉfano, Y. DE (1971): Ecologie des espèces du genre Pisidium Pf. (Mollusques, Sphaeriidac) dans les étangs du Carlit (Pyrénées). - La Terre et la Vie 25, 96-131, Paris.

Feldmann, R. (1971): Die Kleinmuscheln (Sphaeriidae) des mittleren Ruhrtales. - Decheniana 123, 1/2, 27-47, 2 Abb., 2 Tab., Bonn.

Kuiper, J. G. J. (1968): Die spätpleistozänen Pisidien des ehemaligen Ascherslebener Sees. - Arch. Molluskenk. 98, 1/2, 23-38, 56 Abb., 1 Tab., Frankfurt a. M.

OKLAND, K. A. (1971): On the Ecology of Sphaeriidae in a High Mountain Area in South Norway. Norwegian J. Zool. 19, 2, 133-143, Oslo.

Tetens, A. \& Zeissler, H. (1964): Über das Vorkommen der seltenen Pisidienarten im Norddeutsch-Polnischen Raum sowie im Eder- und Schwalmgebiet von Hessen, nebst ökologischen Angaben und Beobachtungen. - Malakol. Abh. staatl. Mus. Tierkunde Dresden 1, 5, 89-133, Dresden.

Zeissler, H. (1971): Die Muschel Pisidium. Bestimmungstabelle für die mitteleuropäischen Sphaeriaceae. - Limnologica (Berlin) 8, 2, 453-503, Berlin.

Manuskript eingeg. 17. 4. 1975.

Anschrift des Verf.: Dr. Claus Meier-Brook, D 7403 Ammerbuch 4, Sommergasse 10. 